\title{
Block-based Compressive Sensing Image Fusion Method Based on Particle Swarm Optimization Algorithm
}

\author{
Xianhu $\mathrm{Li}^{1 \text {, a }}$, Jingguo $\mathrm{Lv}^{2, \mathrm{~b}}$, Shan Jiang ${ }^{3, \mathrm{c}}$, and Xin Pan ${ }^{4, \mathrm{~d}}$ \\ ${ }^{1,2,3,4}$ Beijing University of Civil Engineering and Architecture.Beijing, 102616, China.

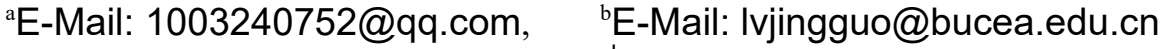 \\ 'E-mail: 379768614@qq.com, dE-Mail: 1457353243@qq.com
}

\begin{abstract}
Keywords: Compressive sensing, Particle swarm optimization, Fusion coefficient, self-adaptability Abstract. This In order to solve the problem that the spatial matching is difficult and the spectral distortion is large in traditional pixel-level image fusion algorithm. In this paper, we proposed an block-based compressive sensing image fusion method based on particle swarm optimization algorithm. We get the compressive measurements of input images by block-based compressive sensing (BCS) and fused them with the rule of linear weighting, while the fusion coefficients $\omega\left(\omega_{1}, \omega_{2}\right.$, $\omega_{3} \ldots, \omega_{\mathrm{n}}, \mathrm{n}$ is the divided number of blocks of the image to be fused) of each block were selected by particle swarm optimization algorithm. In the iterative process, the image fusion coefficient $\omega_{i}$ is taken as particle, and the optimal value is obtained by combining the optimal objective function, taking the coefficient $\omega_{\mathrm{i}}$ as the weight value. The algorithm ensures the optimal selection of fusion effect with a certain degree of self-adaptability. To evaluate the fused images, this paper uses five kinds of index parameters such as Entropy, Standard Deviation, Average Gradient, Degree of Distortion and Peak Signal-to-Noise Ratio. The experimental results show that the image fusion effect of the algorithm in this paper is better than that of traditional methods.
\end{abstract}

\section{Introduction}

Image fusion is an optimization method for spatial information and spectral information, which eliminates suppression and redundant information, highlights the information needed, and provides a more targeted and accurate images in various research fields [1]. Currently, the fusion methods include weighted average fusion, HSI transform algorithm, Brovey transform, principal component analysis algorithm, Gram-Schmidt Spectral Sharpening, Pan-Sharpening, wavelet fusion algorithm etc.[2]. In recent years, some scholars have proposed the fusion methods based on compressive sensing (CS) [3]. Alin Achim (2008) proposed a new image fusion algorithm in the compressive domain by using an improved sampling pattern, the study demonstrates that CS-based image fusion has a number of perceived advantages in comparison with image fusion in the multi-resolution (MR) domain [4]. Yang (2015) proposed an image fusion method based on block-based compressed sensing (BCS) that can reduce the storage requirements and reconstruction cost to a certain extent [5]. In this paper, we proposed an block-based compressive sensing image fusion method based on particle swarm optimization algorithm. With the block-based compressive sensing, we can take the advantage of small number of sampling and small memory requirement and fast reconstruction and simple fusion decision, which is very beneficial to the fusion of big remote sensing images, while the particle swarm optimization algorithm has the advantage of getting global optimization value while calculating the coefficients of each bock of the images to be fused. In the iterative process of particle swarm optimization algorithm, the image fusion coefficient $\omega i$ is used as the particle, and the information entropy and the average gradient function are used as the optimization objective function. The goal is to get $\omega(\omega 1, \omega 2, \omega 3 \ldots, \omega n$,$) , which can maximize the value of functions of entropy and$ the average gradient. Then we take this optimal value as a weight coefficient during the use of weighted fusion rules for image fusion. This method can not only ensure the optimization of the fusion effect, but also make the fusion algorithm have a certain degree of adaptability.

\section{Compressive Sensing(CS)}

Candes and Donoho put forward CS theory in 2006 [6]. And then it had a rapid development on the bases of the existing theories such as signal reconciliation analysis, time-frequency analysis, 
statistical probability theory, matrix analysis, functional analysis, statistical probability theory and so on [7]. Compressive Sensing can achieve compression at the same time as sampling.

Compressive sensing explores the essential structure of the signal from the overall situation, which can get rid of the links between signal frequency and other physical measurement. As long as the signal has a compressible sparse domain, it can be linearly projected onto a low-dimension observation vectors using a measurement matrix that is non-coherent with the transformation matrix. The sample value in projection space contains enough information. So we can use the sparse optimization theory to accurately reconstruct the original signal from a small number of sample values with high probability.

The theory of compression sensing mainly includes the sparse representation of the signal, the measurement matrix design and reconstruction algorithm selection. One of the advantages is that it gathers the steps of data collection and data sample into one. The data is compressed at the same time as the signal acquisition, and the cost in the signal acquisition and processing is greatly reduced..

The superiority of CS is that the sampling quantity is far less than the amount of data obtained by the traditional Nyquist sampling method, breaking through the limitation of the Nyquist sampling theorem.

\section{The BCS Image Fusion Method Based on Particle Swarm Optimization Algorithm}

In this paper, we proposed an block-based compressive sensing image fusion method based on particle swarm optimization algorithm by combining the advantages of block-based compressive sensing that it needs small number of sampling and has little memory required and fast reconstruction with simple fusion decision, and the Particle Swarm Optimization Algorithm that can get global optimization value while calculating the coefficients of each blocks of the images to be fused. The particle swarm optimization algorithm is used to determine the image fusion weight coefficient $\omega$ in the proposed method, which can avoids the problem that the experience value used in traditional methods can't be adjusted adaptively with different characteristics of different images. In this method, the particle swarm optimization algorithm is used to select the fusion coefficients $\omega$ $\left(\omega_{1}, \omega_{2}, \omega_{3}, \cdots, \omega_{n}\right)$. In the iterative process, the image fusion coefficient $\omega_{i}(i=1,2,3 \cdots n)$ is used as particle; the functions of information entropy and the average gradient are taken as the optimization objective function, which goal is to get the maximum values of the information entropy and the average gradient. So we can get the optimal value under the defined optimization objective function. The optimal value is taken as the target fusion weight coefficient used in the weighted fusion rule in block-based compressive sensing image fusion. The method can guarantee the optimization of the fusion effect and make the fusion method have a certain degree of adaptability.

The process of BCS image fusion based on Particle swarm optimization (PSO) is as follows:

Step 1: Raw image processing: The high resolution image is meshed with the multi-spectral image, we can obtain two images with high spatial information matching. Then reshape the image according to the specific research area.

Step 2: The Multi-spectral image and the Panchromatic image are respectively divided into blocks (In this experiment, the original image is divided into 6 rows and 6 columns by means of uniform block method).

Step 3: Then we get the measurement matrix $y_{i}^{\prime}$ and $y_{i}$ of Multi-spectral image and the Panchromatic image got in step 2 by compressive sensing algorithm.

Step 4: The image measurements are fused: $y^{\prime}=\omega y_{1}+(1-\omega) y, \omega \in(0,1)$;

Step 5: The particle swarm optimization algorithm is used to determine the image fusion weight coefficient $\omega$. In the iterative process, the image fusion coefficient $\omega_{i}(i=1,2,3 \cdots n)$ are used as particles; the functions of information entropy and the average gradient are taken as the optimization objective function, which goal is to get the maximum values of the information entropy and the average gradient

Step 6: Reconstruct the image from the fused compressive measurements by OMP (orthogonal matching tracking), and matched it with the original Multi-spectral image, then we finally get the fusion image. 
The process of block-based compressive sensing image fusion based on the particle swarm optimization algorithm is as shown in Figure 1:

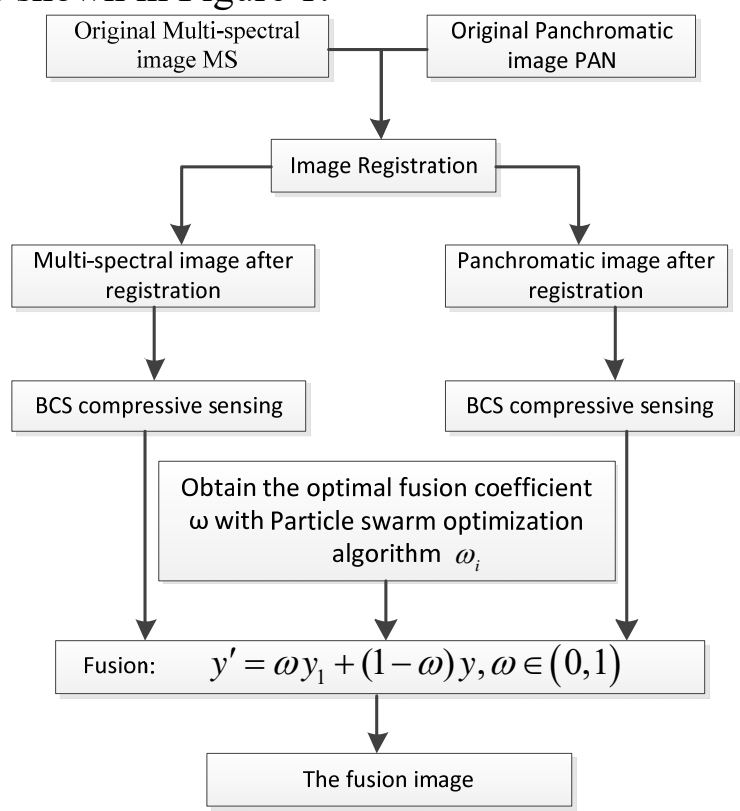

Fig1. The flow chart of BCS image fusion algorithm based on particle swarm optimization algorithm In this paper, the algorithm is validated by using the combination of WorldView panchromatic image and multi-spectral image, and then compared with the fusion effect of traditional methods, such as HIS transformation, PCA fusion, Compressive sensing image fusion, non-adaptive HIS-CS image fusion. The fusion results of different methods are as shown in Figure 4:

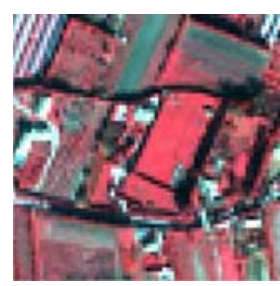

(a) Original Image

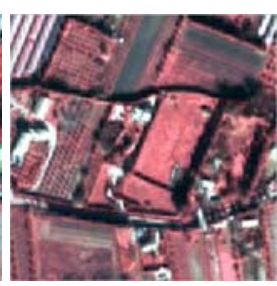

(b) PCA

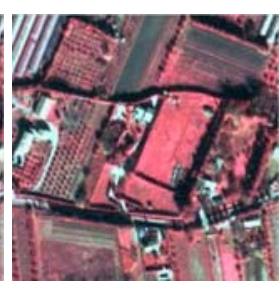

(c) HIS

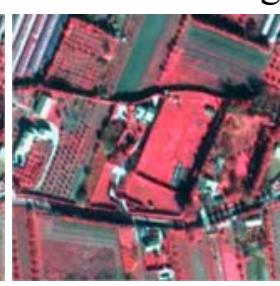

(d) CS
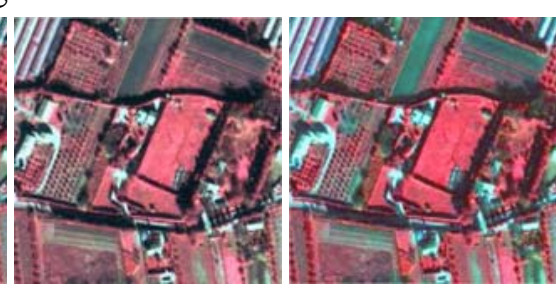

(e) HIS-C (f) this paper method

Fig 2. The results of different fusion methods

\section{Evaluation and Analysis of Image Fusion Results}

After By comparing the fusion image of different methods of Figure 4, we can find out that there is obvious improvement of the spatial resolution of each method, but there are differences between the improvement degrees of various algorithms. The common feature of the fusion images is that the spectral information has been changed, but the methods of this paper and the CS image fusion have the smallest spectral changes, the color information is mostly consistent with the original images. So in terms of spectral information retention, the methods of this paper and CS image fusion is better than the traditional methods. But the method of this paper has a better spatial resolution improvement than the method of CS image fusion.

Table 1. The Comparison of various evaluation indicators with different fusion methods

\begin{tabular}{l|l|l|l|l|l}
\hline & AG & EN & DD & PSNR & STD \\
\hline PCA & 6.8018 & 11.1301 & 55.9768 & 21.5463 & 50.5000 \\
HIS & 8.8623 & 13.1488 & 53.3584 & 21.9316 & 57.0659 \\
CS & 6.2396 & 12.5802 & 33.6010 & 20.7519 & 57.4108 \\
HIS-CS & 8.8269 & 10.5890 & 53.2103 & 20.7950 & 56.7286 \\
Proposed method & 9.3711 & 13.8277 & 44.3443 & 25.0350 & 57.7704 \\
\hline
\end{tabular}

The image fusion effect can be adjust by different evaluation indicators, such as Average Gradient (AG), Entropy (EN), Degree of Distortion (DD), Peak Signal to Noise Ratio(PSNR), Standard Deviation (SD) et.al.. The result of different image evaluation indicators is shown as in table 1. As we 
can see from data of Table 1, the value of AG, EN, PSNR, STD of the method in this paper are larger than that of other methods, so the method in this paper has the optimal evaluation indicators. The value of DD of this method is larger than CS image fusion, but is smaller than the other traditional methods. So we can see that the spectral information retention of the proposed method is better than the other traditional methods, but not as well as CS image fusion. Overall, the proposed method has a better performance than the traditional methods.

\section{Conclusion}

The In this paper, particle swarm optimization algorithm is used to improve the block-based compressive sensing image fusion algorithm. In the proposed method, we combine a series of fusion weight coefficients $\omega$ as particle swarm, and find the optimal value among them to be the optimal fusion weight coefficient $\omega$, which can make the proposed method have certain self-adaptability. In addition, by controlling the step size, we can get a more optimized result, which can balance the improvement of the spatial resolution and spectral information retention. So that by adjusting the step size, we can make the results to meet our requirements. Overall, the proposed method can improve the fusion effect to a certain extent.

But the proposed method has shortcomings ether. The proposed method performs slightly worse in spectral information retention than that of CS image fusion, though it has shown a great advantages in subjective and objective evaluation indices. In addition, in order to make the fusion weight coefficients $\omega$ more optimal, it will lead to a large computation by setting the step size too small. This needs to be further optimized and improved in the lasting research.

\section{References}

[1] Wang X. Research on Remote Sensing Image Fusion Method Based on Compressed Sensing[D]. Beijing : China University of Geosciences, Beijing, 2015.

[2] Yang J. Research on Image Fusion Method Based on Wavelet Transform [D].Beijing : China University of Geosciences, Beijing, 2014.

[3] Guo J. Research on Satellite Remote Sensing Image Fusion Method Based on Compressive Sensing Theory [D]. Beijing: Beijing Jiaotong University, 2013.

[4] Wan T, Canagarajah N, Achim A, et al. Compressive image fusion[C]. international conference on image processing, 2008: 1308-1311.

[5] Senlin Yang, Guobin Wan, Jinghuai Gao. Remote sensing image fusion based on block compressive Sensing [J]. Application Research of Computers, 2015, 32(01): 316-320.

[6] Donoho D L. Compressed sensing[J]. IEEE Transactions on Information Theory, 2006, 52(4):1289-1306.

[7] Gesen Zhang. Research on Compressive Sensing and Some Application Techniques[D]. Harbin : Harbin Engineering University, 2012. 